\title{
Dor Crônica, Depressão, Saúde Geral e Suporte Social em Pacientes Fibromiálgicos e Oncológicos
}

\author{
Chronic Pain, Depression, General Health and Social Support in Fibromyalgic and \\ Oncological Patients
}

\section{Dolor Crónico, Depresión, Salud General y Soporte Social en Pacientes Fibromiálgicos y Oncológicos}

\author{
Cátia Regina Messias \\ Felipe Augusto Cunha \\ Gabriela da Silva Cremasco ${ }^{1}$ \\ Makilim Nunes Baptista \\ Universidade São Francisco (USF)
}

\begin{abstract}
Resumo
O objetivo do estudo foi verificar a associação entre sintomatologia depressiva e saúde geral, dor crônica e percepção do suporte social em 80 mulheres, com idades entre 31 e 82 anos $(M=53,86 ; D P=12,68)$, com fibromialgia e/ou câncer. Entre os principais resultados, verificou-se que a sintomatologia depressiva e os outros construtos investigados estiveram associados entre si. Os pacientes com sintomatologia depressiva apresentaram maior quantidade de pontos dolorosos e maior frequência e intensidade de dor, demonstrando uma correlação de magnitude fraca e positiva $(r=0,37)$. Observou-se também uma correlação de magnitude fraca $(r=-0,20)$ entre a EBADEP-HOSP$A M B$ e EPSUS, indicando que, quanto mais sintomas depressivos, menor a percepção do suporte social recebido. Por fim, os resultados do estudo mostraram que o nível de dor interfere diretamente na qualidade de vida do sujeito, podendo ser um fator que estimula o desenvolvimento de condições psicossociais, tais como a depressão e menor percepção do suporte social.
\end{abstract}

Palavras-chave: qualidade de vida, dor, fibromialgia, câncer

Abstract
The objective of the study was to verify the association between depressive symptomatology and general health, chronic pain, and social support perception in 80 women, aged between 31 and 82 years $(M=53.86, S D=12.68)$, with fibromyalgia and/or cancer. Among the main results, it was verified that the depressive symptomatology and the other investigated constructs were associated with each other. Patients with depressive symptomatology presented more pain points and greater frequency and intensity of pain, showing a weak and positive magnitude correlation ( $r=0.37)$. There was also a weak magnitude correlation ( $r=-0.20$ ) between EBADEP-HOSP-AMB and EPSUS, indicating that the more depressive symptoms, the lower the perceived social support. Finally, the results of the study showed that the level of pain directly interferes with the quality of life of the subject and can be a factor that stimulates the development of psychosocial conditions, such as depression and lower perception of social support.

Keywords: quality of life, pain, fibromyalgia, cancer

\section{Resumen}

El objetivo del estudio fue verificar la asociación entre sintomatología depresiva y salud general, dolor crónico y percepción del soporte social en 80 mujeres, con edades entre 31 y 82 años ( $M=53,86$, DP $=12,68)$, con fibromialgia y/o cáncer. Entre los principales resultados, se verificó que la sintomatología depresiva y los otros constructos investigados estuvieron asociados entre sí. Los pacientes con sintomatología depresiva presentaron mayor cantidad de puntos dolorosos y mayor frecuencia e intensidad de dolor, demostrando una correlación de magnitud débil y positiva $(r=0,37)$. Se observó también una correlación de magnitud débil $(r=-0,20)$ entre EBADEP-HOSP-AMB y EPSUS, indicando que, cuanto más síntomas depresivos, menor la percepción del soporte social recibido. Por último, los resultados del estudio mostraron que el nivel de dolor interfiere directamente en la calidad de vida del sujeto, pudiendo ser un factor que estimula el desarrollo de condiciones psicosociales, tales como la depresión y menor percepción del soporte social.

Palabras clave: calidad de vida, dolor, fibromialgia, cáncer

${ }^{1}$ Endereço de contato: Rua Waldemar César da Silveira, 105, Jardim Cura D’ars, Campinas, SP, Brasil, CEP 13045-510. E-mail: gabisilva10@hotmail.com 


\section{Introdução}

A dor é definida, conforme a Associação Internacional para o Estudo da Dor (Merskey, 1986), como relacionada a um dano tecidual atual ou potencial e caracterizada por experiências sensoriais e emocionais desagradáveis. Santos, Pereira, Resende, Magno e Aguiar (2006) relataram que a dor é entendida como uma experiência sensorial complexa e que se altera a partir da memória, expectativas e emoções, além de se tratar de uma evidência de alterações da integridade física e/ou emocional das pessoas.

A dor crônica afeta os aspectos físico, psicológico e social dos pacientes, além de gerar prejuízos em diferentes âmbitos da sua vida, ocasionando preocupações, sentimento de incapacidade, incertezas e medos na vida dessas pessoas (Loduca et al., 2014). Entre os grupos de pacientes com maiores queixas de dor crônica, há aqueles com fibromialgia e câncer. $A$ fibromialgia, também chamada de síndrome fibromiálgica musculoesquelética (SFM), referese a uma das doenças reumáticas mais comuns, sendo caracterizada principalmente por dores musculoesqueléticas difusas e crônicas (Heymann et al., 2010). Já o câncer corresponde ao crescimento desordenado de células que atingem órgãos e tecidos e que pode se expandir para outras áreas do corpo, sendo responsável por $5 \%$ dos casos de dor crônica. Trata-se de um problema de saúde pública de nível mundial, com predomínio de cânceres de pulmão, mama, próstata e cólon nos países desenvolvidos, e cânceres de estômago, fígado, cavidade oral e colo do útero nos países em desenvolvimento (Instituto Nacional de Câncer [INCA], 2016).

A prevenção da dor se faz importante, e sua evolução deve ser constantemente monitorada, de modo a proporcionar intervenções adequadas para o seu controle (Jales, 2009). A ausência de tratamento adequado para a dor e o sofrimento psíquico do paciente podem desencadear uma expressão de sofrimento mais intensa do que o induzido pela patologia orgânica de base, resultando em uma maior possibilidade de insucesso em seu tratamento (Salazar \& Motta, 2013). Outros fatores relacionados à baixa adesão ao tratamento foram discutidos por Souza et al. (2014), tais como os efeitos colaterais dos remédios, crenças relacionadas ao tratamento, problemas na comunicação com a equipe de saúde e baixa percepção de suporte social. Em relação ao suporte social especificamente, Pedroso e Sbardelloto (2008) discorreram que ele pode auxiliar na adesão do paciente com dor crônica ao tratamento, funcionando como um aspecto importante para o enfrentamento e a adaptação diante de doenças.

É importante frisar a necessidade de avaliar a presença e severidade da dor a partir do relato do paciente, levando-se em conta o significado que a dor tem para ele, suas fantasias e medos em relação à progressão da doença. Tendo em vista que nem sempre a dor crônica é controlável, o paciente passa a ter de conviver com ela e com as limitações decorrentes, sendo que os pacientes que demonstram maior aceitação tendem a relatar menos sintomas depressivos, por exemplo (McCracken, 1998).

Indivíduos com dor crônica comumente apresentam alguma comorbidade psíquica, principalmente a depressão, ocasionando em incapacidade, além de impactos na qualidade de vida, convívio familiar e social. Um exemplo é o estudo de Wilson et al. (2009), em que os participantes que tinham dor moderada a extrema tinham até duas vezes mais chance de apresentar sintomas de depressão $(\chi 2=5,89, p=0,015)$ e quase três vezes mais ansiedade $(\chi 2=15,43, p<0,001)$. 
Buscando verificar a prevalência de sintomatologia depressiva em pacientes com fibromialgia e sua associação com qualidade de vida, Berber, Kupek e Berber (2005) realizaram um estudo. Participaram 70 pacientes, que responderam ao General Health Questionnaire (GHQ-28) e ao Medical Outcome Short Form Health Survey (SF-36). Os resultados indicaram depressão leve em 32,9\% da amostra, depressão moderada em $21,4 \%$ e depressão severa em $12,9 \%$. Além disso, a depressão se relacionou positivamente com a diminuição da qualidade de vida em relação às escalas de condicionamento físico, funcionalidade física, percepção da dor, funcionalidade social, saúde mental, funcionalidade emocional e percepção da saúde geral.

Ferreira et al. (2015) avaliaram possíveis sintomas de depressão em um grupo específico de mulheres com dores crônicas no joelho, para observar se essas dores tendem a interferir na qualidade de vida desses indivíduos e aumentar as chances de desenvolver depressão. Participaram 75 mulheres, com média de 67 anos, sendo 40 com diagnóstico de dores crônicas nos joelhos e 35 sem a presença de dor. Foram aplicados o Inventário de Depressão de Beck (BDI) e SF-36. As mulheres com dores no joelho pontuaram mais sintomas de depressão (57,5\%), além de apresentar qualidade de vida inferior $(58,2 \%)$ comparada com o grupo sem relato de dor.

Tojal e Costa (2014) conduziram também um estudo a respeito do impacto do câncer de mama na avaliação da depressão e percepção do suporte social. A pesquisa contou com 150 mulheres diagnosticadas com câncer, com idades entre 20 e 79 anos, que responderam à Escala Reduzida de Ajustamento ao Câncer, o SF-36 e o BDI. Os principais resultados demonstraram que, à medida que as mulheres apresentaram maior idade (mais de 50 anos), maior foi a possibilidade de terem visões mais negativas e fatais sobre a doença. Verificou-se também que, quanto maior a percepção do suporte social, menor é o descontentamento da pessoa com a sua doença e maiores as chances de demonstrar pensamentos positivos de enfrentamento.

O estudo de Santos, Garcia, Pacheco, Vieira e Santos (2014) teve como objetivo avaliar a qualidade de vida, dor e depressão em pacientes com câncer de reto. Foram selecionados e avaliados 88 pacientes; destes, somente 41 sobreviveram à operação e, por fim, foram convocados à avaliação final. Os instrumentos utilizados para avaliar a qualidade de vida foram o European Organisation for Research and Treatment of Cancer (EORTC), Colorectal Cancer-Specific Quality of Life Questionnaire Module (QLQ-C30) e o Colorectal Cancer-specific Quality of Life Questionnaire Module (QLQ-CR38); para a intensidade da dor, utilizou-se a Escala Visual Analógica e Escala de Estado Geral de Saúde; e, para depressão, utilizou-se o BDI. Os principais resultados demonstraram que $52 \%$ dos pacientes apresentaram dor e $47 \%$ tiveram sintomas de depressão. Houve também correlação positiva forte entre intensidade dolorosa e depressão $(r=0,71)$, além de correlação negativa moderada entre depressão e qualidade de vida $(r=-0,45)$.

A partir dos estudos supracitados, observa-se como a dor crônica tende a influenciar diretamente no desenvolvimento de alguns transtornos mentais, tais como a depressão e na percepção do suporte social. Nesse sentido, o presente estudo teve como objetivo avaliar a associação entre sintomatologia depressiva e saúde geral, dor crônica e percepção do suporte social em mulheres com fibromialgia e câncer. 


\section{Método}

\section{Participantes}

Participaram 80 mulheres, com idades entre 31 e 82 anos $(M=53,86 ; D P=12,68)$, sendo a maioria casadas (70\%) e viúvas (15\%), de quatro instituições de saúde (um ambulatório de oncologia, um ambulatório de especialidades médicas e duas clínicas de dor) de três cidades do interior do estado de São Paulo. Em relação ao diagnóstico, 31 (38,8\%) delas afirmaram ter o diagnóstico de câncer; nove (11,3\%), câncer e outras dores crônicas; 12 (15,0\%), síndrome fibromiálgica musculoesquelética (SFM); cinco (6,3\%) apresentaram SFM com câncer juntos; oito (10,0\%), SFM e outras dores crônicas; e o grupo de dor crônica diversa totalizou $15(18,8 \%)$ mulheres.

\section{Instrumentos}

Questionário Sociodemográfico/Saúde: composto por questões referentes a sexo, idade, além de diagnóstico de depressão.

Escala Baptista de Depressão (Versão Hospital-Ambulatório) - (EBADEP-HOSP-AMB) (Baptista, 2013): trata-se de um instrumento de rastreio de sintomas da depressão, sendo direcionado a amostras ambulatoriais/hospitalares. A escala, com sua versão preliminar, foi constituída por 43 itens, em escala do tipo Likert de três pontos, com pontuação mínima de zero e máxima de 86 pontos. O instrumento foi construído a partir de indicadores sintomáticos de depressão, com exceção dos descritores vegetativos/somáticos. Os indicadores foram provenientes das teorias cognitiva (Beck, Rush, Shaw, \& Emery, 1997) e comportamental (Ferster, Culbertson, \& Boren, 1977), além dos manuais internacionais de diagnóstico da Associação Americana de Psiquiatria, DSM-IV-TR (APA, 2002), e da Organização Mundial de Saúde, CID-10 (OMS, 1993). No estudo de Cremasco e Baptista (2018), foram verificadas as propriedades psicométricas do instrumento, que apresentou índices de fidedignidade adequados, tanto para o fator $1(0,95)$ quanto para o fator $2(0,89)$, com variância total explicada de $56 \%$.

Questionário de Dor McGill (Br-MPQ) (Melzack, 1975): o instrumento adaptado para uso no Brasil por Pimenta e Teixeira (1996) tem como objetivo obter medidas qualitativas de dor e sua intensidade. Avalia as qualidades sensoriais, afetivas, temporais e miscelânea da dor. Apresenta uma avaliação da distribuição espacial, tendo quatro grupos. O primeiro deles é o sensitivo-discriminativo, que se refere às propriedades mecânicas, térmicas, de vividez e espaciais da dor; afetivo-motivacional, que corresponde à dimensão afetiva de aspectos de tensão, medo e respostas neurovegetativas; cognitivo-avaliativo, que possibilita ao paciente indicar a avaliação global da experiência dolorosa; e miscelânea, o qual consiste na compilação de diversos descritores de dor que não se encaixam nos outros grupos. O questionário contém ainda uma escala de intensidade que varia de 0 a 5, um diagrama corporal para representação do local da dor e caracterização da periodicidade e duração da queixa álgica. A consistência teste-reteste foi de 0,94.

Questionário de Saúde Geral de Goldberg - (QSG) (Goldberg, 1972): o instrumento foi adaptado por Pasquali, Gouveia, Andriola, Miranda e Ramos (1994) e tem como objetivo identificar a gravidade de distúrbios psicológicos. É composto de 60 itens, em uma escala do 
tipo Likert de quatro pontos, contendo cinco fatores, sendo esses o Estresse Psíquico, Desejo de Morte, Falta de Confiança na Própria Capacidade de Desempenho, Dísturbios do Sono e Dísturbios Psicossomáticos. O índice de confiabilidade para a pontuação total do instrumento foi de 0,95 .

Escala de Percepção de Suporte Social (Versão Adulto) - (EPSUS-A) (Cardoso \& Baptista, 2014): instrumento que tem por objetivo avaliar a satisfação com o suporte social recebido. É composta por 36 itens, em escala do tipo Likert de quatro pontos, com pontuação variando entre zero e 108, divididos em quatro fatores, denominados de Afetivo, Interações Sociais, Instrumental e Enfrentamento de Problemas. O instrumento foi desenvolvido a partir do conceito de suporte social de Rodriguez e Cohen (1998). O índice de confiabilidade por meio do alfa de Cronbach foi de 0,92 para o fator Afetivo, 0,75 para Interações Sociais, 0,82 no fator Instrumental, alfa de 0,83 para Enfrentamento de Problemas. A confiabilidade total do instrumento foi de 0,94 .

\section{Procedimentos}

Após autorização das instituições e da aprovação do projeto pelo Comitê de Ética em Pesquisa da Universidade São Francisco, sob protocolo de número 365.350, as participantes assinaram o Termo de Consentimento Livre e Esclarecido (TCLE). Os instrumentos foram aplicados de forma individual, tendo como primeiro instrumento o Questionário Sociodemográfico/Saúde, seguido do Questionário de Dor McGill (Br-MPO), da Escala Baptista de Depressão (Versão Hospital-Ambulatório) - EBADEP-HOSP-AMB, do Questionário de Saúde Geral de Goldberg (QSG) e da Escala de Percepção de Suporte Social (EPSUS-A). O tempo de aplicação foi de, aproximadamente, uma hora e trinta minutos.

\section{Análise de Dados}

Os dados foram analisados por meio do programa SPSS (Statistical Package for the Social Sciences - versão 21.0), sendo adotado o nível de significância de 5\%. Para verificar as correlações entre as escalas, foi utilizado o Teste de Correlação de Pearson, segundo a classificação proposta por Dancey e Reidy (2006); e, para a análise de possíveis diferenças de médias, foi utilizado o Teste $t$ de Student para dois grupos.

\section{Resultados}

Os resultados referentes à intensidade da dor demonstraram que $43(53,8 \%)$ das participantes classificaram a dor como moderada; $28(35,4 \%)$, como intensa; e oito $(10,8 \%)$, como leve. Quanto à frequência semanal de dor, $51(63,8 \%)$ participantes sentem a dor por sete dias da semana, enquanto sete $(8,8 \%)$ sentem por três dias na semana. Quanto à classificação de intensidade atual da dor, 28 (35\%) participantes disseram ser desconfortante; 22 (27,5\%), horrível; e 20 (25\%), angustiante. Em se tratando do humor, 35 (43,8\%) relataram apresentar diagnóstico de depressão e 28 (35\%) faziam uso de medicamentos para depressão.

Foram verificadas as correlações entre os instrumentos, sendo encontrada uma magnitude fraca e positiva $(r=0,37 ; p<0,05)$ entre a quantidade de pontos dolorosos do McGill e a EBADEP-HOSP-AMB, indicando que, quanto maior a presença de pontos dolorosos, 
maior a sintomatologia depressiva. Verificou-se magnitude moderada e positiva entre a EBADEP-HOSP-AMB e os fatores do QSG, que variaram entre $(r=0,49 ; p<0,05)$ e $(r=0,64 ; p$ $<0,05)$, com exceção do fator Distúrbio do Sono, que apresentou magnitude fraca $(r=0,39$; $p<0,05)$, sugerindo que, à medida que os sintomas de depressão aumentam, maior se torna a presença de estresse psíquico, desejo de morte, presença de distúrbio psicossomático, distúrbio do sono e falta de confiança na própria capacidade DE desempenho. As correlações entre a EBADEP-HOSP-AMB e as dimensões da EPSUS variaram entre $(r=-0,48 ; p<0,05)$ e $(r$ $=-0,62 ; p<0,05)$, com exceção da dimensão Instrumental, que apresentou magnitude fraca $(r=-0,20 ; p<0,05)$, indicando que, quanto mais sintomas depressivos, menor a percepção do suporte social recebido.

É válido dizer que a estatística inferencial utilizada neste trabalho considerou todos os diagnósticos como um só grupo, tendo em vista que, independentemente dos diagnósticos, os grupos que inicialmente foram separados (Grupo de Câncer, Grupo de SFM, Grupo de Dor Crônica Diversa, SFM com Câncer Juntos, SFM e Outras Dores Crônicas e Câncer e Outras Dores Crônicas) não diferenciaram as principais variáveis relacionadas com a dor. Assim, foi conduzida uma análise para verificar possíveis diferenças de média entre o grupo de pessoas que relataram diagnóstico de depressão e o grupo que indicou não ter o diagnóstico em relação à pontuação nas escalas de depressão, saúde geral e suporte social. Os resultados obtidos podem ser verificados na Tabela 1.

Tabela 1

Diferenças de média entre grupo que relatou ter diagnóstico de depressão $(N=35)$ e grupo que relatou não ter diagnóstico $(N=45)$

\begin{tabular}{|c|c|c|c|c|c|}
\hline Instrumento & $\begin{array}{l}\text { Diagnóstico } \\
\text { de Depressão }\end{array}$ & Média & DP & $\mathbf{t}$ & $\mathbf{P}$ \\
\hline EBADEP-HOSP-AMB & $\begin{array}{l}\text { Não } \\
\text { Sim }\end{array}$ & $\begin{array}{l}15,62 \\
31,42\end{array}$ & $\begin{array}{l}11,51 \\
14,71\end{array}$ & $-5,39$ & 0,001 \\
\hline EPSUS- Afetivo & $\begin{array}{l}\text { Não } \\
\text { Sim }\end{array}$ & $\begin{array}{l}41,65 \\
32,14\end{array}$ & $\begin{array}{c}9,80 \\
13,62\end{array}$ & 3,47 & 0,001 \\
\hline EPSUS- Interações Sociais & $\begin{array}{l}\text { Não } \\
\text { Sim }\end{array}$ & $\begin{array}{l}9,15 \\
7,05\end{array}$ & $\begin{array}{l}4,07 \\
3,92\end{array}$ & 2,32 & 0,023 \\
\hline EPSUS- Instrumental & $\begin{array}{l}\text { Não } \\
\text { Sim }\end{array}$ & $\begin{array}{l}13,93 \\
12,57\end{array}$ & $\begin{array}{l}5,74 \\
5,41\end{array}$ & 1,07 & 0,284 \\
\hline EPSUS- Enfrentamento de Problemas & $\begin{array}{l}\text { Não } \\
\text { Sim }\end{array}$ & $\begin{array}{l}15,64 \\
12,31\end{array}$ & $\begin{array}{l}4,36 \\
6,75\end{array}$ & 2,53 & 0,014 \\
\hline EPSUS- Total & $\begin{array}{l}\text { Não } \\
\text { Sim }\end{array}$ & $\begin{array}{l}80,45 \\
64,08\end{array}$ & $\begin{array}{l}20,74 \\
26,53\end{array}$ & 2,99 & 0,004 \\
\hline QSG-Estresse Psíquico & $\begin{array}{l}\text { Não } \\
\text { Sim }\end{array}$ & $\begin{array}{l}25,24 \\
33,00\end{array}$ & $\begin{array}{l}13,15 \\
12,55\end{array}$ & $-2,66$ & 0,009 \\
\hline QSG-Desejo de Morte & $\begin{array}{l}\text { Não } \\
\text { Sim }\end{array}$ & $\begin{array}{l}10,62 \\
14,08\end{array}$ & $\begin{array}{l}6,93 \\
8,42\end{array}$ & $-2,01$ & 0,047 \\
\hline $\begin{array}{l}\text { QSG- Desconfiança na própria } \\
\text { capacidade de desempenho }\end{array}$ & $\begin{array}{l}\text { Não } \\
\text { Sim }\end{array}$ & $\begin{array}{l}37,24 \\
43,94\end{array}$ & $\begin{array}{l}12,89 \\
13,94\end{array}$ & $-2,22$ & 0,029 \\
\hline
\end{tabular}




\begin{tabular}{lccccc}
\hline \multicolumn{1}{c}{ Instrumento } & $\begin{array}{c}\text { Diagnóstico } \\
\text { de Depressão }\end{array}$ & Média & DP & t & P \\
\hline QSG- Distúrbio do Sono & Não & 11,08 & 5,90 & $-2,81$ & 0,006 \\
& Sim & 15,31 & 7,50 & & \\
QSG- Distúrbio Psicossomático & Não & 21,62 & 8,18 & $-2,12$ & 0,037 \\
& Sim & 25,48 & 7,89 & & \\
QSG- Saúde Geral & Não & 105,82 & 42,92 & $-2,79$ & 0,007 \\
\hline
\end{tabular}

Como pode ser observado na Tabela 1, as diferenças entre os grupos foram significativas em relação às dimensões e pontuação total dos instrumentos, com exceção à dimensão Instrumental da EPSUS. De modo geral, as pessoas que indicaram ter diagnóstico de depressão pontuaram mais na EBADEP-HOSP-AMB, apresentando mais sintomas de depressivos do que as sem diagnóstico relatado. Além disso, aquelas sem diagnóstico apresentaram também uma maior percepção de suporte social, bem como maior índice de saúde geral.

\section{Discussão}

O presente estudo teve como objetivo avaliar associação entre sintomatologia depressiva com saúde geral, dor crônica e percepção do suporte social. A partir dos resultados encontrados, foi possível perceber que a maioria dos participantes demonstraram sentir dores com intensidades moderadas e com alta frequência, aproximadamente todos os dias. Além disso, várias pacientes relataram ter diagnóstico de depressão e algumas faziam uso de medicamentos psicotrópicos para regular os sintomas depressivos. Segundo Loduca et al. (2014), é comum que pessoas que sofrem com algum tipo de doença crônica sejam afetadas em diversos âmbitos, podendo acarretar prejuízos psicológicos e desenvolver um possível quadro depressivo.

Segundo os resultados provenientes das correlações, foi possível observar que, entre o Questionário de Dor McGill e a EBADEP-HOSP-AMB, foi obtida uma correlação estatisticamente significativa e com magnitude fraca. Espera-se que, quanto mais pontos corporais de dor, maior o nível da sintomatologia depressiva, podendo afetar a qualidade de vida do indivíduo (Wilson et al., 2009). Ferreira et al. (2015) comentaram que, ao passo que os pacientes se queixam de dores, principalmente as que são consideradas do tipo crônica, que são aquelas dores constantes e sem melhora por um longo período, tendem a desenvolver mais problemas emocionais, como depressão e aumento de pensamentos distorcidos sobre a saúde, interferindo diretamente na sua qualidade de vida.

Outra correlação que demonstrou magnitude moderada e positiva foi entre a EBADEPHOSP-AMB e os fatores do QSG, indicando que, ao passo que o nível da depressão tende a aumentar, aumentam também os sintomas mais biológicos, como oscilação no tempo do sono e fadiga motora, piorando a saúde geral do paciente (Pedroso \& Sbardelloto, 2008). Como demonstrado no estudo de Santos et al. (2014), os pacientes com altos níveis de dor também apresentaram mais sintomas de depressão. Finalizando as correlações entre os instrumentos, a EBADEP-HOSP-AMB com as dimensões da EPSUS variou entre correlações de magnitudes fracas a moderadas, demonstrando que, quanto mais a pessoa apresenta um suporte social adequado, menor é a chance de desenvolver depressão. 
Um dos motivos do fenômeno do suporte social ser tão importante é que, quando o sujeito passível de alguma dor crônica tem esse suporte em um bom nível, tende a conseguir aderir aos tratamentos de saúde com mais propriedade, sendo um ponto importante e necessário para um bom enfrentamento da doença (Pedroso \& Sbardelloto, 2008). Tojal e Costa (2014) demonstraram também, a partir de um estudo com pacientes que sofriam com dor crônica proveniente de câncer, que os pacientes que aparentavam ter uma boa percepção do suporte social conseguiam elaborar com mais facilidade estratégias de enfrentamento para a doença e, com isso, minimizar possíveis pensamentos negativos. Cabe considerar que eram esperadas correlações com essas magnitudes, tendo em vista que, apesar de serem construtos relacionados, eles são diferentes.

A partir dos resultados obtidos sobre as diferenças de médias, foi feita uma comparação entre os sujeitos que relataram ter diagnóstico de depressão e os que não apresentaram sintomatologia depressiva. Assim, as diferenças entre esses grupos foram significativas, comparadas com as dimensões dos instrumentos aplicados, com exceção da dimensão Instrumental da EPSUS, que não apresentou média significativa. No geral, os sujeitos com diagnóstico de depressão e com relatos de dores pontuaram com maior frequência na EBADEP-HOSP-AMB, demonstrando mais sintomas depressivos do que as pessoas sem diagnóstico prévio. Os resultados obtidos no presente estudo vão ao encontro da pesquisa de Berber et al. (2005), em que os participantes também apresentaram níveis considerados de depressão, principalmente os sujeitos que se queixaram de algum tipo de dor.

Santos et al. (2014) explicaram que, quando a pessoa começa a apresentar grandes níveis de dores, e isso se torna crônico, aos poucos esse individuo tende a se isolar e viver em busca de tratamentos médicos para os cuidados necessários. A partir desse novo quadro de vida, existe uma grande tendência de o sujeito minimizar suas relações sociais - por exemplo, afastamento do trabalho, do âmbito familiar e de atividades geradoras de prazer -, de forma que isso possa interferir na saúde mental e possivelmente se tornar um preditor do surgimento de possíveis transtornos mentais, tais como a depressão, o estresse e a ansiedade.

Por outro lado, as pessoas que não apresentaram nenhum tipo de diagnóstico, sendo esse de dor e/ou depressão, demonstraram uma melhor percepção de suporte social, interferindo na sua visão de saúde no geral. Santos et al. (2006) discutiram que o suporte social é um fator protetivo para possíveis problemas de saúde, ajudando o indivíduo a preservar suas relações pessoais e, com isso, aumentar chances de ter mais cuidado pessoal quando está em uma situação de vulnerabilidade por algum tipo de doença ou transtorno psicológico.

Diante do exposto, é importante ressaltar que a pesquisa teve algumas limitações durante o seu processo. Primeiramente, o número reduzido de participantes e a divisão dos grupos, sendo que $43,8 \%$ dos sujeitos relataram ter diagnóstico de depressão, porém $35 \%$ faziam uso de medicamento para controlar os sintomas depressivos, o que pode ter enviesado o nível de sintomatologia depressiva no momento de responder à EBADEP-HOSP-AMB. Apesar disso, cabe considerar que, no estudo de Souza et al. (2014), a maior parte da amostra (74,07\%) também fazia uso de antidepressivos, sendo necessárias novas investigações e maior controle quanto a essa variável.

Em pesquisas futuras, é importante avaliar os construtos em amostras mais heterogêneas de pacientes com diferentes tipos de dores crônicas. Apesar das limitações, os objetivos da presente pesquisa foram cumpridos. Ter instrumentos que consigam identificar 
adequadamente a sintomatologia depressiva pode auxiliar no desenvolvimento de intervenções eficazes em pessoas com questões médicas associadas, sendo a EBADEP-HOSP-AMB um instrumento promissor.

\section{Financiamento}

O presente trabalho foi financiado pela Coordenação de Aperfeiçoamento de Pessoal de Nível Superior (Capes) e pelo Conselho Nacional de Desenvolvimento Científico e Tecnológico (CNPq).

\section{Referências}

American Psychiatric Association. (2002). Manual Diagnóstico e Estatístico de Transtornos Mentais DSM-IV-TR (4a ed.). Porto Alegre: Artmed.

Baptista, M. N. (2013). Escala Baptista de Depressão - (Versão Ambulatório) - Ebadep-HospAmb. Relatório Técnico não publicado. Programa de Pós-Graduação Stricto Sensu em Psicologia da Universidade São Francisco, Itatiba, São Paulo.

Beck, A. T., Rush, A. J, Shaw, F. B., \& Emery, E. (1997). Terapia cognitiva da depressão. Porto Alegre: Artes Médicas.

Berber, J. S. S., Kupek, E., \& Berber, S. C. (2005). Prevalência de Depressão e sua Relação com a Qualidade de Vida em Pacientes com Síndrome da Fibromialgia. Revista Brasileira de Reumatologia, 45(2), 47-54. Recuperado de http://www.scielo.br/pdf/rbr/v45n2/ v45n2a01

Cardoso, H. F. \& Baptista, M. N. (2014). Escala de Percepção de Suporte Social (versão Adulto) - Epsus-A. São Paulo: Hogrefe.

Cremasco, G. S. \& Baptista, M. N. (2018). Análise de estrutura interna da Escala Baptista de Depressão Versão Hospital-Ambulatório (Ebadep-Hosp-Amb). Interação em Psicologia, 22(2), 144-154.

Dancey, C. P. \& Reidy, J. (2006). Estatística sem matemática para Psicologia usando SPSS para Windows. Porto Alegre, RS: Artmed.

Ferreira, A. H., Godoy, P. B. G., Oliveira, N. R. C., Diniz, R. A. S., Diniz, R. E. A. S., Padovani, R. C., \& Silva, R. C. B. (2015). Investigação da ansiedade, depressão e qualidade de vida em pacientes portadores de osteortrite no joelho: Um estudo comparativo. Revista Brasileira de Reumatologia, 55(5), 434-438. doi:http://dx.doi.org/10.1016/j.rbr.2015.03.001

Ferster, C. B., Culbertson, S., \& Boren, M. C. (1977). Depressão clínica. In C. B. Ferster, S. Culbertson, \& M. C. Boren (Orgs.), Princípios do Comportamento (M. I. Silva, M. A. Rodrigues, \& M. B. Pardo, trads., pp. 699-725). São Paulo: Hucitec.

Goldberg, D. P. (1972). Questionário de Saúde Geral de Goldberg: Manual técnico QSG. São Paulo: Casa do Psicólogo.

Heymann, R. E., Paiva, E. S., Helfenstein Junior, M., Pollak, D. F., Martinez, J. E., Provenza, J. R., . . . \& Fonseca, M. C. M. (2010). Consenso brasileiro do tratamento da fibromialgia. Revista Brasileira de Reumatologia, 50(1), 56-66. doi:http://dx.doi. org/10.1590/S0482-50042010000100006

Instituto Nacional do Câncer. (2016). Incidência de câncerno Brasil. Brasília: INCA. Recuperado de http://www.inca.gov.br/estimativa/2016/estimativa-2016-v11.pdf 
Jales, L. (2009). Dor no câncer e cuidados paliativos - A importância do controle sobre dor do paciente. Arquivos do go Simpósio Brasileiro e Encontro Internacional sobre a Dor Simbidor, 9, 43-48.

Loduca, A., Müller, B. M., Amaral, R., Souza, A. C. M. S., Focosi, A. S., Samuelian, C., ... \& Batista, M. (2014). Retrato de dores crônicas: percepção da dor através do olhar dos sofredores. Revista Dor, 15(1), 30-35. doi:http://dx.doi.org/10.5935/1806-0013.20140008

McCracken, L. M. (1998). Learning to live with the pain: Acceptance of pain predicts adjustment in persons with chronic pain, Pain, 74(1), 21-27. doi:http://dx.doi.org/10.1016/ S0304-3959(97)00146-2

Melzack, R. (1975). The McGill Pain Questionnaire: Major Properties and Scoring Methods. Pain, 1(3), 277-299. doi:http://dx.doi.org/10.1016/0304-3959(75)90044-5

Merskey, H. (1986). Classification of chronic pain: Description of chronic pain syndromes and definitions of pain terms. Pain, 3, 215-217.

Organização Mundial da Saúde. (1993). Classificação dos transtornos mentais e do comportamento - CID-10: Descrições e diretrizes diagnósticas. Porto Alegre: Artes Médicas.

Pasquali, L., Gouveia, V. V., Andriola, W. B., Miranda, F. J., \& Ramos, A. L. (1994). Questionário de Saúde Geral de Goldberg (QSG): Adaptação Brasileira. Psicologia: Teoria e Pesquisa, 10(3), 421-437.

Pedroso, R. S., \& Sbardelotto, G. (2008). Qualidade de Vida e Suporte Social em Pacientes Renais Crônicos: Revisão Teórica. Psicópio: Revista Virtual de Psicologia Hospitalar e da Saúde, 7, 32-35.

Pimenta, C. A. M., \& Teixeira, M. J. (1996). Questionário de Dor McGill: Proposta de adaptação para a língua portuguesa. Revista Escola de Enfermagem da USP, 30(3), 473483. doi:http://dx.doi.org/10.1590/S0080-62341996000300009

Rodriguez, M. S. \& Cohen, S. (1998). Social Support. Encyclopedia of mental health. New York: Academic Press.

Salazar, O. M., \& Motta, N. W. (2013). Tumores Avançados - Epidemiologia, Etiopatogenia, Diagnóstico e Estadiamento Clínico. Recuperado de http://www1.inca.gov.br/pqrt/ download/tec_int/cap3.pdf

Santos, L. J. F., Garcia, J. B. S., Pacheco, J. S., Vieira, E. B. M., \& Santos, A. M. (2014). Quality of life, pain, anxiety and depression in patients surgically treated with cancer of rectum. Arquivos Brasileiros de Cirurgia Diagnóstica, 27(2), 96-100. doi: http://dx.doi.org/10.1590/ S0102-67202014000200003

Santos, C. C., Pereira, L. S. M., Resende, M. A., Magno, F., \& Aguiar, V. (2006). Aplicação da versão brasileira do questionário de dor McGill em idosos com dor crônica. Acta Fisiátrica, 13(2), 75-82. Recuperado de http://www.revistas.usp.br/actafisiatrica/article/ view/102586/100864

Souza, B. F., Moraes, J. A., Inocenti, A., Santos, M. A., Silva, A. E. B. C., \& Miasso, A. I. (2014). Mulheres com câncer de mama em uso de quimioterápicos: Sintomas depressivos e adesão ao tratamento. Revista Latino-Americana de Enfermagem, 22(5), 866-873. doi:http://dx.doi.org/10.1590/0104-1169.3564.249

Tojal, C. \& Costa, R. (2014). Ajustamento mental ao cancro da mama: Papel da depressão e suporte social. Revista Psicologia, Saúde e Doenças, 15(3), 777-789. doi:http://dx.doi. org/10.15309/14psd150317 
Wilson, K. G., Chochinov, H. M., Allard, P., Chary, S., Gagnon, P. R., Macmillan, K. . . . \& Fainsinger, R. L. (2009). Prevalence and correlates of pain in the Canadian National Palliative Care Survey. Pain Research \& Management, 14(5), 365-370. doi:http://dx.doi. org/10.1155/2009/251239

Recebido em: 25/09/2018

Última revisão: 07/06/2019

Aceite final: 02/07/2019

\section{Sobre os autores:}

Cátia Regina Messias: Mestre pelo Programa de Pós-Graduação Stricto Sensu em Psicologia com ênfase em Avaliação Psicológica, pela Universidade São Francisco (USF). Psicóloga. E-mail: catiamessias. psico@gmail.com Orcid: https://orcid.org/0000-0002-4264-9700

Felipe Augusto Cunha: Doutorando e mestre pelo Programa de Pós-Graduação Stricto Sensu em Psicologia com ênfase em Avaliação Psicológica, pela Universidade São Francisco (USF). Bolsista pela Coordenação de Aperfeiçoamento de Pessoal de Nível Superior (CAPES). Psicólogo pela USF. E-mail: flpcunha@terra.com.br, Orcid: https://orcid.org/0000-0001-7977-9712

Gabriela da Silva Cremasco: Doutoranda e mestre pelo Programa de Pós-Graduação Stricto Sensu em Psicologia com ênfase em Avaliação Psicológica, pela Universidade São Francisco (USF). Bolsista pela Coordenação de Aperfeiçoamento de Pessoal de Nível Superior (CAPES). Psicóloga pela USF. E-mail: gabisilva10@hotmail.com, Orcid: https://orcid.org/0000-0003-2075-8049

Makilim Nunes Baptista: Doutor pelo Departamento de Psiquiatria e Psicologia Médica da Universidade Federal de São Paulo (USP). Psicólogo. Professor do Programa de Pós-Graduação Stricto Sensu em Psicologia da Universidade São Francisco (USF) e bolsista de produtividade pelo Conselho Nacional de Desenvolvimento Científico e Tecnológico (CNPq).E-mail: makilim01@gmail.com, Orcid: https://orcid.org/0000-0001-6519-254X 
